\title{
L'écriture et la survie. Sur une mélancolie
}

Jean-Marie Barnaud

\section{OpenEdition}

Journals

Édition électronique

URL : http://journals.openedition.org/ccs/463

DOI : $10.4000 /$ ccs.463

ISSN : 2558-782X

Éditeur :

Presses universitaires de Rennes, Association des lecteurs de Claude Simon

Édition imprimée

Date de publication : 31 décembre 2006

Pagination : $17-30$

ISBN : 9782914518895

ISSN : 1774-9425

Référence électronique

Jean-Marie Barnaud, «L'écriture et la survie. Sur une mélancolie », Cahiers Claude Simon [En ligne], 2 | 2006, mis en ligne le 20 septembre 2017, consulté le 15 septembre 2020. URL : http://

journals.openedition.org/ccs/463 


\section{L'écriture et la survie Sur une mélancolie par Jean-Marie BARNAUD}

[...] et à la fin il dit Mélancolie, le journaliste s'exclamant Mélancolie !..., le dévisageant de derrière les verres sans montures de ses lunettes de docteur, les sourcils levés, de cet air à nouveau sceptique, réprobateur, irrité presque...

Le Jardin des Plantes

Le journaliste, tête bien carrée, mène son enquête psychologique avec obstination, il est celui qui veut comprendre, et qui a les armes pour ; on ne lui en conte pas, à celui-là, il juge et réprouve, c'est la raison qui le mène, une raison tout hygiène, comme sa chemise qui sort de chez le blanchisseur ; son apparence plaide pour l'ordre et le savoir, « lunettes de docteur ${ }^{1}$ ", lunettes dernier cri, comme du reste sa montre suisse, qui obsède Simon, puisqu'il revient souvent sur ces cadrans dont les aiguilles " tourn[ent] en rond [...] [c]omme si le temps n'avançait pas, tournait sur lui-même " $(J P, 82)$.

${ }^{1}$ Le Jardin des Plantes, Minuit, 1997, p. 299 (désormais JP). 
Donc le journaliste veut comprendre, et en particulier deux choses : comment on fait " pour vivre avec la peur " $(J P, 35)$ en temps de guerre et sur le champ de bataille, c'est sa première obsession. Et puis pourquoi le cavalier Simon a suivi son colonel sur une route ouverte au feu des snipers, risquant sa mort, alors que l'officier le laissait libre de partir.

À cette seconde question, Simon répond d'abord de façon abrupte que c'est « $[\mathrm{m}]$ écaniquement ou par vanité, ou par stupidité ou par simple fatigue » $(J P, 292)$, loin d'avancer une "fadaise » à la Stendhal (Julien Sorel éprouverait la " sensation délicieuse " de " marcher au grand air " alors qu'il monte à la guillotine [297]), avant de préciser qu'il serait de toute façon resté par fidélité à sa parole. Sinon (cette fois-ci, le ton est plutôt stendhalien...) : «De quoi aurais-je eu l'air? " (292).

Mais la réponse ne convainc pas l'enquêteur qui a tenté sans succès d'expliquer l'obstination suicidaire du cavalier par " [le] désespoir, [le] renoncement, [l'] abdication » $(J P, 299) \ldots$

Et c'est alors que Simon dit le mot "Mélancolie »- avec une majuscule -, ce qui entraîne la réprobation outragée que l'on sait.

Mais il le dit, avec quelle réticence et comme en désespoir de cause, "à la fin" : une fin qui n'est pas seulement celle de l'entretien, mais qui annonce celle du livre. Peut-être aussi celle de l'œuvre, son aboutissement... Il le dit après hésitation, après un long silence, une interruption, au cours de laquelle il aura perçu "ce même indifférent et menaçant grondement, cette espèce de bruit de fond, cette rumeur étale, sans plus de consistance qu'une faible et unique vibration dans quoi vient se confondre toute l'agitation du dehors, se neutraliser toute la violence, les passions, les désirs, les peines, les terreurs " (JP, 299).

J'aimerais apprendre ce qu'il en est de cette "rumeur ", de cette " unique vibration » et pourquoi c'est après qu'on l'a perçue, ou vécue, que vient aux lèvres le mot Mélancolie... 
I.

Bien sûr, les mots nous piègent, et surtout ceux que continue de porter la culture, chargés d'Histoire et de valeurs; et Mélancolie, de l'aveu de Simon, est " un mot ambigu, à malentendus " (JP, 301), c'est faute de mieux qu'il le risque, sachant de quel amas de sens et de cendre il va brouiller l'entretien, quelles images il véhicule, depuis l'ange de Dürer jusqu'aux " mièvres » préraphaélites (302), des poses et lamentations romantiques au rejet décadent de la culture elle-même.

Pourtant il le risque.

Et l'écrit encore ici, dans l'après-coup de l'entretien, alors qu'il construit Le Jardin des Plantes, et qu'affleurent par moments dans le texte, non pas les soupirs de la lamentation, bien sûr, encore moins la mièvrerie, mais, à l'évocation du passage et du travail du temps, le sentiment classiquement mélancolique de la perte nécessaire de toute chose - toute chose destinée à « être jeté[e] au rebut »-, l'évidence d'un effondrement programmé : par exemple, à propos des Ghosts Cities, le temps comme "monstre invisible, indolent, sans hâte et vorace " qui efface " peu à peu jusqu'au souvenir de toute vie » $(J P, 69-70)$; ou, depuis le corps malade, la mémoire de la santé d'avant : " avec cette différence qu'alors, sur votre cheval [...] votre corps était plein de vie " (312); ou encore, vu du bord du Nil, le lent passage des bateaux - image connue - « dont on pouvait entendre de loin le tap-tap du moteur grandissant peu à peu, s'approchant, puis tout près, mêlé au bruit de l'eau repoussée par l'étrave, bouillonnant à l'arrière en gros remous » (31-32) ; ou encore tel tableau d'arrière-saison et sa "lumière rousse et mélancolique " (348)...

Cela, le journaliste l'entendrait clairement, cela qui pourtant ne répondrait pas à sa question.

Et donc, que faudrait-il qu'il entende, dans ce mot de Mélancolie, qui ne prête pas à malentendu. À supposer qu'il puisse même l'entendre...

Avec - toujours ! - sa chemise impeccable, et la cravate, et les lunettes, et la montre, que peut-il savoir de l'expérience du soldat à 
la « chemise crasseuse et puante " $(J P, 97)$, qui signifie la peur « collée à votre peau ", mieux qu'aucune glose. "Non Vous ne comprenez pas Vous ne pouvez tout simplement pas C'est absolument impossible".

En vérité, quelque chose lui manque, qui le ferait entrer dans la véritable intelligence de l'expérience de Simon, et mieux : dans sa sympathie; quelque chose que la seule représentation de la peur, de la guerre et de la déroute n'éclairerait pas suffisamment. Que lui manque-t-il ? Ce qui a transformé pour toujours en écrivain Simon le survivant, exigeant de lui qu'il porte fidèlement, comme une énigme, la vérité - justesse et justice - de cet événement : il a vu sa mort en face. Le journaliste, non.

Je souligne ce verbe : qu'il porte...

C'est que - on me pardonnera ce détour - je vis depuis quelque temps dans la compagnie de Béliers, ce grand livre que Derrida consacre, en hommage à Gadamer, au commentaire d'un poème de Celan, et qui ouvre à une méditation sans fin sur les rapports de l'écriture et de la survie, et sur l'origine de ce que Derrida nomme une " mélancolie sans âge ", écrivant notamment de certains événements que leur leçon " peut demeurer une vérité essentielle et permanente, mais cela peut aussi arriver une seule fois, singulièrement, dans une histoire, et cette occurrence serait alors contresignée dans un récit comme un événement et confiée à quelqu'un ${ }^{2}$ ".

L'événement que Le Jardin des Plantes confie à notre sympathie, on le sait bien, c'est la scène inaugurale dont la brûlure efficace se redit et se répète dans ce livre après La Route des Flandres : "[...] le seul véritable traumatisme qu'il est conscient d'avoir subi et à la suite duquel sans aucun doute son psychisme et son comportement général dans la vie se trouvèrent profondément modifiés fut, comme il a essayé de le raconter, ce qu'il éprouva pendant l'heure durant laquelle il suivit ce colonel [...] avec la certitude d'être tué dans la seconde qui allait suivre. " $(J P, 223)$.

Ce moment exceptionnel fonde une épokhè, pour reprendre le

2 Jacques Derrida, Béliers, Editions Galilée, 2003, p. 73. 
mot de Derrida ${ }^{3}$, qui « retient le souffle, suspend le jugement ou la conclusion ", vous tient "bouche bée ", le temps que s'accomplisse la nécessité, dans sa vérité toujours indéchiffrable, soit, ici, la mort du colonel (et son double certain, la fin du cavalier qui voit sa propre mort en face) que Simon représente, en un cliché instantané, sous la forme théâtrale et dérisoire d'un geste chevaleresque suspendant la scène un instant dans l'ouvert du ciel, avant la chute : "Par un curieux réflexe, au moment où il est atteint par la rafale, il dégaine et élève son sabre à bout de bras : le cheval et le cavalier tombent ainsi sur le côté, d'un bloc. » $(J P, 72)$.

Or, lorsque l'on sait (" certitude ") que, "d'une minute à l'autre on ne sera plus qu'une de ces choses effroyablement immobiles qui ressembl[ent] à des sacs de sciure " ( $J P, 298-299)$, on n'existe pas, non, dans l'exaltation romanesque d'un Julien Sorel, même si l'on a oublié, comme lui, la peur qui paralyse ; la «perspective imminente d'une mort violente » (305) - c'est celle de Dostoïevski au cours du "simulacre d'exécution " qu'il a vécu - fait vivre le condamné dans la conscience panique d'une présence du monde, mais c'est un monde, "qui va continuer d'exister alors que dans quelques instants lui-même ne sera plus rien. "Un comble de désir que la nécessité réactive d'autant plus qu'elle l'aura détruit, bientôt.

Désir panique, ai-je dit... Mais c’est à son propos que, précisément, Simon court le risque d'en appeler à la Mélancolie; il a écrit, c'est moi qui souligne : "déchirante et mélancolique avidité " (JP, 305).

La mélancolie ne chante pas la résignation, elle n'est pas figure de la lamentation ni du ressentiment. Elle inspire à l'âme la plus juste parole pour dire ce suspens exceptionnel du temps où la conscience de la perte est d'une autre nature que celle qu'on reçoit de l'écoulement banal des jours, ou même à l'occasion de maladies, comme celles que Le Jardin des Plantes par trois fois évoque. Elle aussi, pour emprunter encore à Derrida, "mélancolie sans âge " parce que l'événement qui la décide décide d'un rapport au monde qu'aucun dévoilement, aucun récit, aucune représentation des secrets de la

3 Ibid., p. 10. 
petite histoire personnelle ne sauraient circonscrire, elle aussi, donc, inspire un "cogito de l'adieu ", dont le futur antérieur exprime par excellence l'évidence, cruauté et joie confondues : dans quelques instants, le monde et moi aurons été séparés...

Même si, évidemment, je détourne irrévérencieusement le texte de Béliers, puisque ce cogito de l'adieu n'identifie plus ici le destin d'une amitié d'hommes (c'est en effet à propos de son amitié avec Gadamer que Derrida invente ce concept). Mais il me semble qu'il y a, dans l'amour du monde, quelque chose aussi d'une amitié du monde, puisque celui-ci dispense en retour l'évidence de sa splendeur énigmatique à qui a conscience de devoir le perdre, et qui proteste, " furieux, bâillonné, mais hurlant ": " Jamais je n'avais tant désiré vivre, jamais je n'avais regardé avec autant d'avidité, d'émerveillement, le ciel, les nuages, les prés, les haies...» $(J P, 303)$.

Mais c'est aussi une amitié dont ma mort m'aura exclu le premier : autre certitude en quoi réside toute la différence, car il n'y aura plus, dès lors, du monde à moi, de moi au monde, rien ni personne pour rien porter...

Sauf qu'au dernier moment, par un détour ironique, une pitrerie du destin, la mort peut manquer, et faire de celui qu'elle épargne un survivant $t^{4}$.

À jamais cependant, et pour reprendre encore les termes de Simon, l'attente et le face-à-face (et par ailleurs n'est-ce pas aussi toujours sa propre mort qui se montre et fait signe dans celle des autres) auront profondément "[modifié] son psychisme et son comportement général dans la vie " (JP, 223).

Manquerait encore de saisir les arcanes de la scène plus vaste dont cette aventure fut et demeure le jouet, de cette "vague et facétieuse entité (l'Histoire ?), impersonnelle, stupide et impitoyable » (JP, 336).

\footnotetext{
${ }^{4}$ Sur le «survivant ", on sait aussi que Derrida a beaucoup écrit.
} 
Dans Le Jardin des Plantes - dont, en écho au titre même, le bestiaire métaphorique est si riche et si constant - Simon multiplie, à l'occasion des portraits que par fragments il donne des grands acteurs de la guerre où il s'est trouvé pris, Churchill ou Rommel par exemple, les situations où se manifeste ce mixte de grandeur épique et de dérisoire à quoi pour lui elle se résume, et ses acteurs, et l'Histoire en général.

Et le monde lui-même, dont l'épiphanie n'est jamais de pure splendeur, mais du même absurde mélange. Pas étonnant que ses références littéraires soient celles que Flaubert avant lui a déjà convoquées pour caractériser ce qu'il nomme, dans une lettre à Louise Colet, "le grotesque triste ": Shakespeare et Cervantès (à ces deux noms, du reste, Simon ajoute « la Bovary »...) : «Et S. dit [...] que les événements les plus tragiques ont souvent un côté dérisoire et que ce dérisoire même en augmente le tragique voir Cervantès, Shakespeare "mon royaume pour un cheval", sans parler de la Bovary...» (JP, 260).

Grotesque triste... Rien ne rend mieux compte de la belle formule que ce passage du Jardin qui met en scène l'entrecroisement improbable du destin grandiose de Rommel et de celui de Simon, le cavalier obscur, sur la même route des Flandres, tous deux noués inconscients à la beauté du monde, et confondus en elle, mais tous deux acteurs floués, comme le prédisent et le constatent le futur simple puis le futur antérieur et, abruptement et par ironie, du moins s'agissant de Rommel, la chute du texte :

Il fera très beau et le soleil printanier brillera sur les prés, les haies d'aubépine en fleurs, les vergers, teintant de saumon les fumées qui çà et là s'élèveront parfois de véhicules achevant de se consumer, étincelant sur les toits d'ardoise des fermes. Rommel sera passé là au cours de la nuit à la tête de sa division et, sur sa lancée, aura continué tout droit sur Avesnes, Landrecies et Le Cateau, entamant le parcours jalonné de victoires qui le conduira quatre ans plus tard à croquer une pastille de cyanure. $(J P, 160)$

"Les événements les plus tragiques "? Il ne s’agit pourtant pas de plaindre le malheur, ce récit n'est pas une tragédie, ni l'écriture une 
catharsis. Il s'agit de donner corps à cette sorte de regret poignant et d'autant plus poignant qu'on est toujours plus désireux d'étreindre la réalité rugueuse - que le fracas du monde ne soit pas à la hauteur des splendeurs simples que ce monde dispense. Non, pas le malheur, qui se prête si bien à la plainte et au ressentiment; pas de terreur ni de pitié, mais, plus tenace, plus chantant à l'intérieur, et si propre à la mélancolie, quelque chose comme un chagrin.

Comme je trouve signifiant que Simon, dans le Jardin, cite deux fois, et, la seconde, de façon abrupte, sans autre commentaire, comme un leitmotiv ou un programme, cette phrase de Proust à son éditeur: "Remplacer le mot malheur par le mot chagrin et laisser la phrase pareille " $\left(J P, 153^{5}\right)$.

\section{III}

Laisser la phrase pareille. Mais quelle phrase?

Si l'écriture est la seule survie, la seule fidélité possibles, elle expose à une autre épreuve encore, antinomique, celle de sa propre impossibilité : témoigner en vérité ? "C'est impossible en parlant et en écrivant ".

Là prend racine une autre source de la mélancolie simonienne, je la dirai mélancolie seconde, au sens où elle succède à l'événement, à l'épokhè qui cependant la fonde : c'est cette épreuve de l'aporie à quoi mène la tentative de donner forme à la part la plus secrète, insaisissable, et la plus vive cependant, de son aventure individuelle.

La réalité vécue est incommensurable à la parole : mis à la question par qui prétend comprendre, comme ce journaliste, on a vite fait, de réticences en dérobades, d'expérimenter et de prouver à quel point toute réponse est une tricherie. Du point de vue de l'objectivité, laquelle est cependant un leurre, mieux vaudrait s'en tenir à ce simple constat : «[...] dire non pas que $j$ 'avais fait la guerre mais que je m'étais simplement trouvé dedans comme on peut se trouver pris

\footnotetext{
5 Voir aussi p. 138.
} 
dans un orage ou dans un cataclysme et qu'encore ce n'étaient pas les mots (orage, cataclysme) justes [...] et voilà. » $(J P, 77-78)$.

Et voilà, façon cavalière de clore le débat, sauf à lâcher, faute de mieux, comme pour se débarrasser de l'importun, par provocation peut-être, Mélancolie, laissant entendre, dans l'interdit du silence qui vient après, que tout reste à faire néanmoins, interminablement...

Écrire, oui, c'est faire l'essai ( il a essayé de raconter ») d'un récit impossible ; mais cette impasse ne saurait se masquer derrière des formes littéraires dont la rhétorique obligée enfermerait dans un discours ; c'est inventer au contraire l'incomplétude, la fragmentation, la répétition, le tournoiement infini de la phrase autour de ce qui lui fait défaut : on comprend que Simon ait mis en exergue au Jardin des Plantes une citation des Essais, puisqu'en effet, de notre vie, nous ne "délibérons qu'à parcelles ", et qu'il y cite Conrad, à l'entrée de la troisième partie : "Non, c'est impossible : il est impossible de communiquer la sensation vivante d'aucune époque donnée de son existence [...] sa subtile et pénétrante essence. ».

Voilà pourquoi cette œuvre me renvoie constamment à la poésie, s'il est vrai que le récit y est sans cesse interrompu, ou disjoint, par le sentiment d'une incohérence, ou mieux d'une illisibilité du monde, au sein duquel cependant se manifestent, comme autant d'énigmes, des présences, visages du réel que la parole recueille, auxquels elle donne un abri précaire, comme le fait un poème, c'est-àdire avec le sentiment que ce qui surgit là, dans l'obscure théorie des jours et des frêles bavardages des hommes, passe à nouveau incommensurablement les pouvoirs de l'écriture. Il restera toujours quelque chose à dire, qui manque, et qui cependant en appelle à la parole ${ }^{6} \ldots$

\footnotetext{
6 " Die Welt ist fort, ich muss dich tragen " : c'est le vers de Celan que Derrida commente longuement dans Béliers, faisant briller le prisme de plusieurs interprétations ou, mieux, comme il le dit lui-même, d'une " lecture-écriture disséminale " (il est extrait d'un poème dont le bestiaire métaphorique et cosmique donne à ce livre son titre : Widders/bélier). Dans Renverse du souffle (Le Seuil, "La librairie du XX $\mathrm{XX}^{\mathrm{e}}$ siècle ", p. 113) Jean-Pierre Lefebvre le traduit ainsi : "Le monde est parti, il faut que je te porte ». Parmi les pistes ouvertes par Derrida, celle-ci (p. 72 sq.): « Le je dois (ich muss) doit nécessairement se tour-
} 
C'est parfois pur poème en effet - disposition insolite dans la page, effacement des copules qui tiennent d'ordinaire le discours que ces interruptions, comme dans l'évocation de celle qui

se baignait nue dans le lac aux eaux glaciales rides concentriques à partir de ses jambes ses cuisses son corps à la surface polie miroir joncs près des rives cercles d'argent allant s'élargissant sur le reflet noir laqué des bois profonds le silence seulement le bruit frais des gerbes d'eau rejaillissant quand elle s'y plonge un oiseau quelque part

chapelet de gouttelettes qui s'égrenaient tombaient de ses coudes lorsqu'elle ressortait levait les bras pour tordre ses cheveux $(J P, 42)$

Mais ce peut être aussi le tableau "réaliste " d'une scène ou d'un instantané - portrait d'hommes ou de villes, flambées d'érotisme, paysages de ciels vus d'avion, multiples passages d'oiseaux, comme ces "grandes mouettes qui volaient bas silencieusement dans le demi-jour de la nuit arctique " $(J P, 121)$, simples éclats de lumière, simple feuille qu'on " regarde avec étonnement tournoyer sur elle-

ner vers l'avenir. Il s'oriente dans la pensée, comme dirait Kant, vers l'orient de ce qui vient, reste à venir, se lève ou s'élève dans le ciel. " Ainsi tragen, qui "se dit aussi couramment de l'expérience qui consiste à porter un enfant encore à naître ", "parle le langage de la naissance ». Voilà qui trace aussi du moins dans la perspective depuis laquelle j'observe le texte de Simon - les pouvoirs et les devoirs de l'écriture, son devenir : "voir sa mort en face " n'implique pas qu'on doive être écrasé par l'effroi devant cette présence qui, cependant, soudainement absente le monde. Au contraire : d'un point de vue plus général, il s'agit, à partir de là, de porter la nécessité de ce deuil - autrement dit, pour chacun, l'évidence de sa propre mort - en en renversant la donne négative, en y cherchant l'origine de la liberté. Et donc la joie. C'est aussi bien à s'en tenir à cette fidélité à la vie que s'est voué Joë Bousquet, comme l'a si bien montré de son côté Deleuze dans Logique du sens (" de l'événement »). L'épokhè, là encore, enseigne à porter le deuil intime comme on porte un enfant : elle apprend à tourner l'évidence du côté d'une naissance à venir, d'une renaissance, d'une élégance, dont, pour qui écrit, l'écriture est la matière ouverte, la chance, la " liberté libre ", et parfois la grâce. 
même " (276), évocations aussi de supplices et d'exécutions horribles : tous éclats, murmures et fracas, isolés du foisonnement du monde et pour l'évocation desquels Simon peut aussi recourir au plus classique : à la métaphore, à l'équilibre de la phrase... et pourtant ce comble de maitrise, au lieu d'enfermer le lecteur dans sa complétude, de figer son regard, l'ouvre au contraire à nouveau à la conscience de l'énigme :

Quoique l'on entendît tout près le chuintement tumultueux $\mathrm{du}$ torrent qui roulait ses eaux descendues des glaciers personne ne fut autorisé à franchir le grillage pour s'en approcher et il resta invisible, obsédant, comme l'inlassable cavalcade de quelque harde sauvage se bousculant, bondissant, luttant de vitesse avec elle-même, secouant ses crinières.

Le «traumatisme " d'un face-à-face avec sa mort sur la route des Flandres n'a pas seulement décidé d'une origine ; il a conforté celui qui vivait l'expérience, ou l'a confirmé, dans l'évidence d'une distance, d'une séparation irréductibles, antérieures à l'événement : depuis toujours sans doute, ontologiquement, une part de lui, la plus secrète, est étrangère au monde. Voilà qui fonde une solitude, quels que soient par ailleurs les plaisirs et les splendeurs que dispense ce monde. La mélancolie, paradoxalement, n'est pas incompatible avec l'amour de la vie, peut-être même avec la joie : "Déchirante avidité $» \ldots$

La figure de "l'étranger " va bien à Simon, comme le signale par exemple la posture baudelairienne de tel passage du Jardin dans lequel le narrateur se montre - c'est pourtant la guerre et la vie de camp - occupé, le dimanche, à rêver à l'écart, " restant, la tête renversée, à regarder passer les nuages lents au-delà des branches griffues " d'un chêne $(J P, 247-248) .$. . De même plus tard cette manière de n'être plus là soudain, lors d'une réception officielle, de regarder les arbres filtrer la lumière, loin au-delà du brouhaha et des simagrées des invités, cependant que passent en rêve, sur la façade d'un 
"vieux palais couleur de siècles [...] les mélancoliques fantômes de rois et de reines aux cous engoncés de perles ou de fraises » (343)...

D'où ce sentiment d'irréalité parfois, de " demi-somnambulisme ", à l'occasion d'événements précis, comme si un brouillard recouvrait les choses, laissant seulement la pointe aiguë de quelque fait, ou détail, traverser l'épaisseur de la distance, comme " les filets de bave de ce cheval brillant dans le soleil et la grosse croupe rose à la queue pisseuse de l'autre devant moi " : seule présence sensible, dont le cavalier est captif, et d'où lui vient la conscience d'exister au sein d'un monde qui se défait. (Dans Le Tramway, le dernier livre publié, d'une construction très classique cette fois, on retrouvera très vive cette thématique de l'écart, que rend plus poignant encore, et désenchanté, la mélancolie spécifique liée au travail de la mémoire :

Comme si quelque chose de plus que l'été n'en finissait pas d'agoniser dans l'étouffante immobilité de l'air où semblait toujours flotter ce voile en suspension qu'aucun souffle d'air ne chassait, s'affalant lentement, recouvrant d'un uniforme linceul les lauriers touffus, les gazons brûlés par le soleil, les iris fanés et le bassin d'eau croupie sous une impalpable couche de cendres $[\ldots]^{7}$.

La mélancolie travaille en secret l'écriture.

Comme une ligne mélodique rassemble et enveloppe dans un rythme qu'on ne peut confondre les notes disparates étendues sur la portée, elle inspire à qui écrit l'art de tenir ensemble, et sans tricherie, les fragments de réel que recueille la page, gagnant toujours sur l'insignifiance apparente d'une vie, travaillant au dévoilement incertain de sa "subtile et pénétrante essence».

Sans doute l'écriture est-elle la seule résistance à l'absurde : peu à peu, l'essai construit son ordre sur le désordre du monde, ce que Simon observe lui-même : "À partir de là vont simultanément se dérouler plusieurs événements qui, en dépit ou peut-être en raison même de leur apparente incohérence, constituent un tout pratiquement homogène et cohérent » $(J P, 202-203)$.

${ }^{7}$ Le Tramway, Minuit, 2001, p. 141. 
Mais qu'est-ce que l'ordre, celui d'un poème, celui d'un livre?

Sans doute ce que disait Eugène Leroy, à propos de sa peinture : "l'effet obtenu d'éléments désordonnés qui se mettent à chanter juste à l'œil ". La mélancolie, chez Claude Simon, est la mesure secrète d'un tel chant, qui chante juste aussi à l'œil, à l'âme, avec, comme toujours, cette part insaisissable, irréductible à l'analyse, et dont l'impossible capture dirait comment et pourquoi vraiment ça "se met " à chanter juste...

Peut-être est-elle, cette mélancolie, le seul écho fidèle aux beautés et aux joies du monde qui passent, surgissant interdites dans un temps dont elles interrompent la banale circularité, elles qui n'ont d'autre raison que leur seule présence, qui ne reposent sur rien, qui ne disent rien d'autre qu'elles-mêmes, et qui n'attendent rien de nous, sauf notre étonnement, avant de se perdre, elles aussi, dans la "faible et unique vibration dans quoi vient se confondre toute l'agitation du dehors, se neutraliser toute la violence, les passions, les désirs, les peines, les terreurs " $(J P, 299)$.

Annexe : Lettre de Claude Simon à Jean-Marie Barnaud. Cette lettre évoque le texte écrit en réaction au livre de Dominique Viart, Une mémoire inquiète (PUF, 1997), paru en revue en 1998 et envoyé à Claude Simon.

Paris, le 3 mars 1999

\section{Cher Monsieur,}

Pardonnez-moi, tout d'abord, je vous prie, mon retard à répondre à votre aimable lettre et à vous remercier de votre excellent texte adressé à Dominique Viart mais, à mon âge, la moindre grippe met à plat et, comme tout le monde cet hiver, j'y suis passé.

Je n'ai malheureusement pas le temps de vous parler en détail de toutes les intéressantes questions que vous soulevez et auxquelles, travaillant par tâtonnements, je ne pourrais moi-même donner la juste réponse. 
Tout juste pourrais-je me permettre une petite remarque sur l'allusion que vous faites (page 2) aux trois fois où j'ai cru "voir la mort en face " ("maintenant, maintenant, maintenant"). Ce n'est pas tout à fait exact: dans ces trois occasions j'ai pensé (ou non) la mort non pas exactement "en face ", mais annoncée, probable. Autre chose est de la voir exactement en face, c'est-à-dire à peu près certaine dans la seconde à venir, comme je l'ai éprouvé en suivant sur la route de Solre-LeChâteau à Avesnes, ce colonel devenu fou (ou simplement imbécile, puisqu'il était accompagné d'un autre...).

À part ce petit point de détail, toutes vos réflexions sont des plus judicieuses et pertinentes. Mais encore une fois le temps me manque terriblement. Tout au plus puis-je m'arrêter (toujours page 2) sur cette phrase: "Le J. P. tient "pour la première fois" un discours beaucoup plus cohérent sur l'histoire de la seconde guerre mondiale". Vous touchez là à un point, ou plutôt une question, qui me tient à cœur ou, pour parler vulgairement, me reste encore, soixante ans plus tard, sur l'estomac. Parce que (du moins à ma connaissance) un seul historien (le seul Marc Bloch qui, vraisemblablement a payé ce livre de sa vie) a osé écrire sur L'étrange défaite de 1940. Il semble que par un bizarre accord tacite, de même qu'il est, en France, convenu de passer sous silence les honteuses années Pétain, on ait aussi convenu de passer soigneusement sous silence ce qui les avait permises, c'est-à-dire la défaite de mai 40 et en particulier cette très curieuse porte grande ouverte aux Allemands (ou plutôt aux Nazis) par l'État-major français entre Namur et Mézières, et plus particulièrement encore l' "étrange " facilité (80 km en une nuit !...) avec laquelle Rommel a pratiquement encerclé les 43 divisions alliées au nord - d'où Dunkerque (après quoi la bataille était pratiquement terminée*) et Pétain.... [*en fait, elle l'était déjà le 13 au soir lorsque les Allemands ont franchi la Meuse...]

Pardonnez-moi, je vous prie, cette digression fort peu littéraire. Si vous avez des collègues historiens, voilà un beau sujet de recherche (il y a des documents au S. H. A. T, château de Vincennes), et croyez, je vous prie, cher Monsieur, à mes sentiments les plus cordiaux, 